\title{
Cylindrically Periodic Dielectric Waveguide at Submillimeter Waves
}

\author{
N. Llombart", P. H. Siegel \\ California Institute of Technology, Pasadena, CA
}

\section{Introduction}

In the submillimeter and terahertz bands strong inherent dielectric absorption in solids and high skin-depth losses in conductors make it difficult to realize single mode propagating structures with low loss. Traditionally, low loss waveguides in these bands have been realized with oversized hollow cores because the conductivity loss decreases as the propagation constant of the mode approaches that of free space. However this implies that many modes are above cut-off and they may easily be excited, as in case of bends. Another viable candidate to accomplish such a low propagation loss is the high dielectric constant ribbon waveguide [1][2]. Ribbon guide works by maintaining a large ratio between its lateral dimensions (width-to-height) in such a way that the mode propagates on the interface between the dielectric and the air instead of in the material, as in traditional dielectric waveguides. This dramatically lowers the propagation loss of such a mode, however this waveguide has substantial radiation losses at any sharp bends if no coating is used [2]. Waveguides that are cylindrically periodic (CPWG), see Fig. 1, have shown very low loss in the infrared bands [3]. Designs of periodic dielectrics have shown that the mode resembles that of metallic circular waveguide (CWG) [4]. The authors believe that such a waveguide could be the solution for both low propagation loss and low loss around bends.

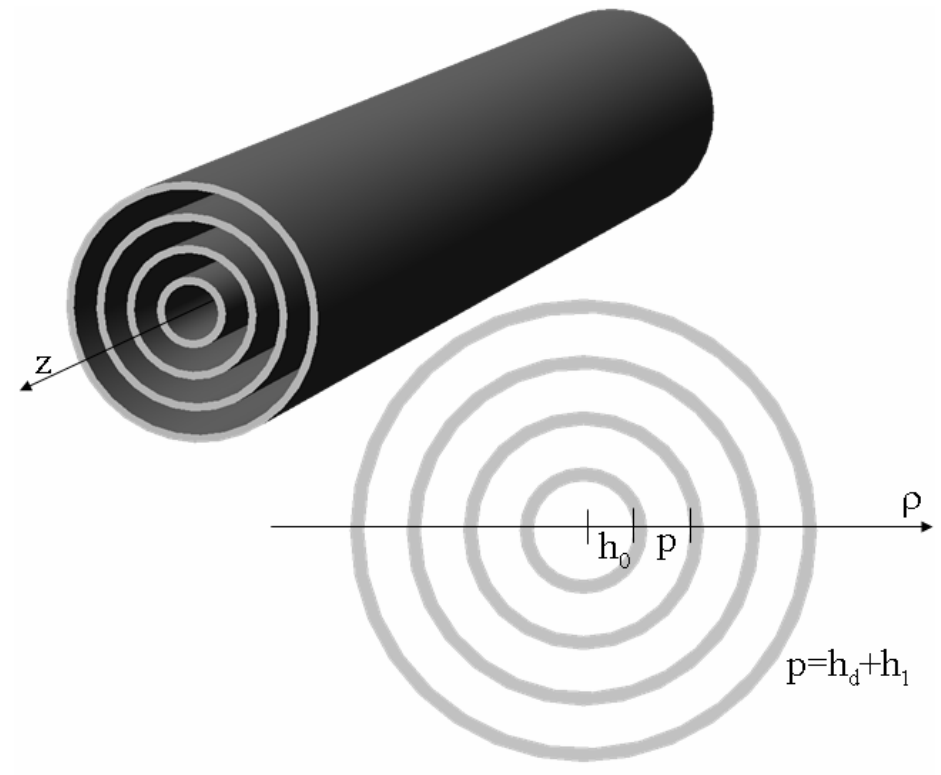

Figure 1: Cylindrically periodic dielectric waveguide (CPWG)

In this work we studied the properties of CPWGs by calculating the leaky wave poles of the pertinent spectral Green's function. The mode distribution can then be obtained by applying the theory of residues. The results are validated with simulations in HFSS. Dielectric and radiation losses are also considered. The latter depends on the dielectric constant and the number of layers. Fabrication of these CPWGs with a large number of layers and high dielectric constant materials is not possible at the present time at these frequencies. Therefore an alternative structure with a small number of layers was also studied, wherein the periodic stratification is terminated at a predetermined position in a metal wall. This eliminates the radiation loss but adds a modest loss contribution from the metal. 


\section{Propagation Constant}

The CPWG has a period of $\mathrm{p}=\mathrm{h}_{\mathrm{d}}+\mathrm{h}_{1}$, where $\mathrm{h}_{\mathrm{d}}$ is a quarter of the wavelength in the dielectric and $h_{1}$ is a quarter of the wavelength in free space, i.e. the same resonant condition used in [5]. The dimension $\mathrm{h}_{0}$ is tuned to resemble the $\mathrm{TE}_{11}$ mode in a conventional CWG. The propagation constant along $\mathrm{z}$ of such a structure can be computed by calculating the zeros of the denominator of the spectral Green's function:

$$
\bar{E}(\rho, \phi, z)=\frac{1}{2 \pi} \sum_{n} e^{j n\left(\phi-\phi^{\prime}\right)} \int_{-\infty}^{\infty} \overline{\bar{G}}_{n}\left(k_{z}, \rho, \rho^{\prime}\right) e^{-j k_{z}\left(z-z^{\prime}\right)} d k_{z}
$$

where $n$ is the index of the Bessel/Hankel functions and the dyad $\overline{\bar{G}}_{n}\left(k_{z}, \rho, \rho^{\prime}\right)$ can be computed by using $2 \times 2$ transmission and reflection matrices as described in [6] or by using $4 \times 4$ matrices as in [7] that ensure the boundary conditions of the tangential fields at the interfaces in $\rho_{i}$.

Figure 2a shows the normalized propagation constant for $n=1$ when 4 dielectric layers of $\varepsilon r=9$ or $\varepsilon r=3.8$ are used. In the same graph the propagation constant of the metallic CWG is also shown. If the stratification is open, there is a radiation loss associated with the imaginary part of the leaky pole, see Fig. $2 b$, where $\mathrm{P}$ indicates the number of dielectric layers.

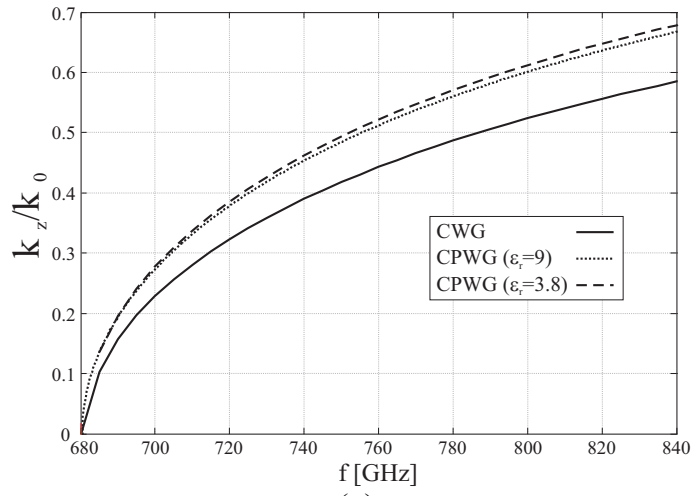

(a)

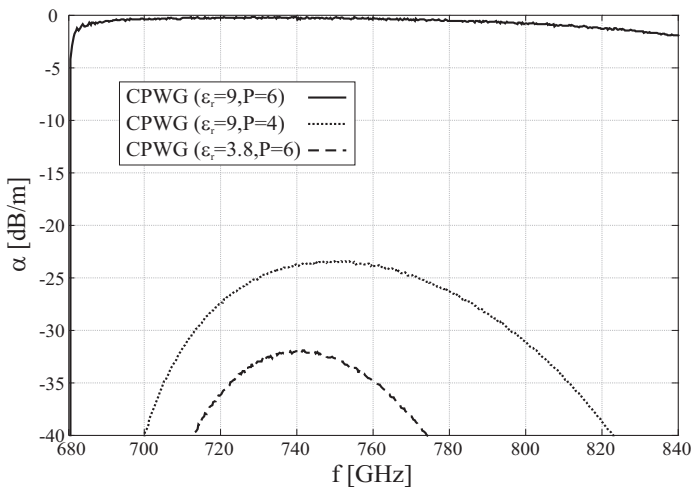

(b)

Fig. 2 Structure dimensions: CWG with $h_{0}=129 \mu \mathrm{m}, \mathrm{CPWG}(\varepsilon \mathrm{r}=9)$ with $\mathrm{h}_{0}=132 \mu \mathrm{m}, \mathrm{h}_{\mathrm{d}}=36.6 \mu \mathrm{m}$ and $\mathrm{h}_{1}=110 \mu \mathrm{m}$ and CPWG $(\varepsilon \mathrm{r}=3.8)$ with $\mathrm{h}_{0}=136.5 \mu \mathrm{m}, \mathrm{h}_{\mathrm{d}}=56.4 \mu \mathrm{m}$ and $\mathrm{h}_{1}=110 \mathrm{~mm}$. (a)

Propagation constant (b) Radiation loss

However this loss can be significantly reduced by increasing the numbers of layers or by bounding the periodic structure by metal instead of air. This latter option will allow fabricating such waveguide with a small number of layers. If the outer layer is terminated with a metal, an additional condition has to be met in order to solve the problem: the tangential electric fields have to be zero at the metal interface. This creates a homogenous equation system, where the condition for a non-trivial solution will determine the dispersion equation of the waveguide modes.

\section{Mode Field Distribution}

In the open structure, once the propagation constant $k_{z}$ is found, the theory of residues can be used to obtain the mode field distribution. The transverse electric field vector 
components are shown in Fig3a at $\mathrm{f}=775 \mathrm{GHz}$. In the core layer they resemble the fields of the CWG as expected from [4]. Figure 2 (b) shows the amplitude of the transverse field.

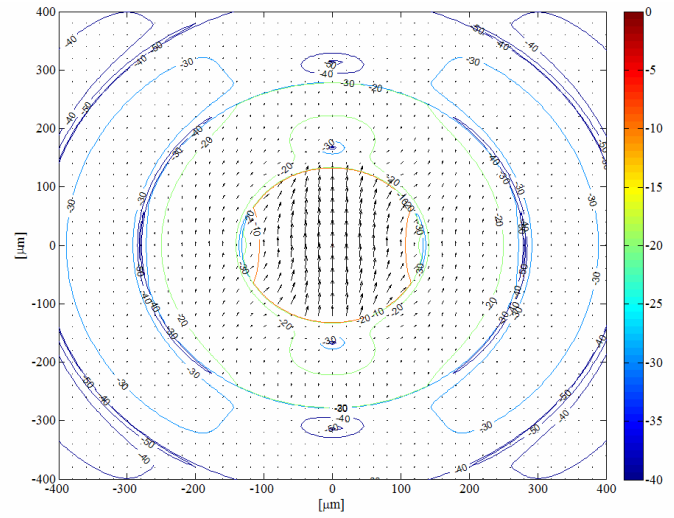

(a)

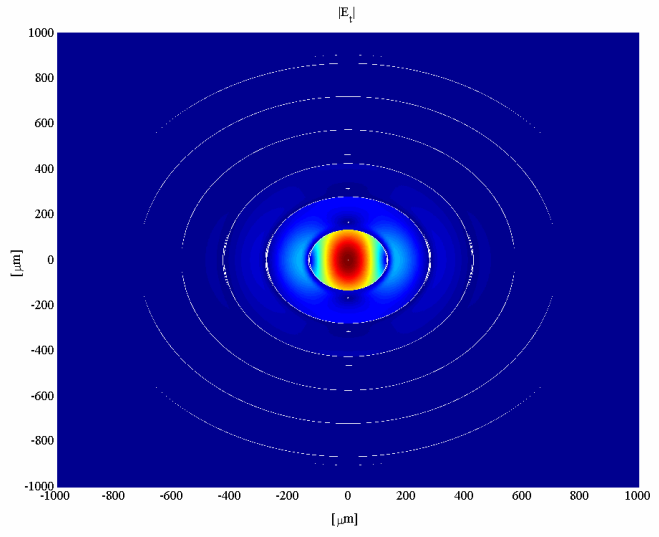

(b)

Fig.3 Vector components (a) and (b) amplitude of the transverse electric field ( $\varepsilon r=9, \mathrm{P}=6$ ).

The electric field for $\phi=0$ is shown in Fig.4a and compared with simulations using HFSS. If a lower dielectric constant is used, i.e. $\varepsilon r=3.8$, the power traveling on the second air layer is increased, as can be seen in Fig.4a. If more layers are used, the field distribution in the already existing layers does not change (see Fig.4b). Moreover, the field distribution in the previous layers remains the same if a metal boundary is placed at the beginning of the next period (since the electric field has a null there). Therefore the propagation constant of Fig. $2 \mathrm{~b}$ also remains the same. In the case of the closed structure, the fields have been obtained by solving the homogenous system with the proper propagation constant.

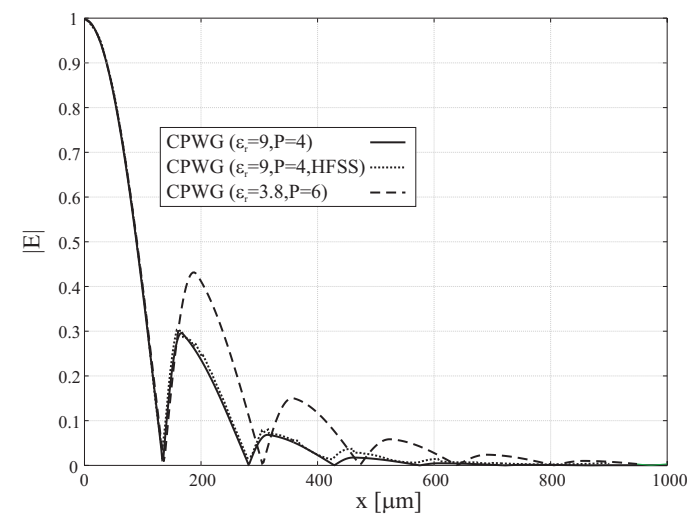

(a)

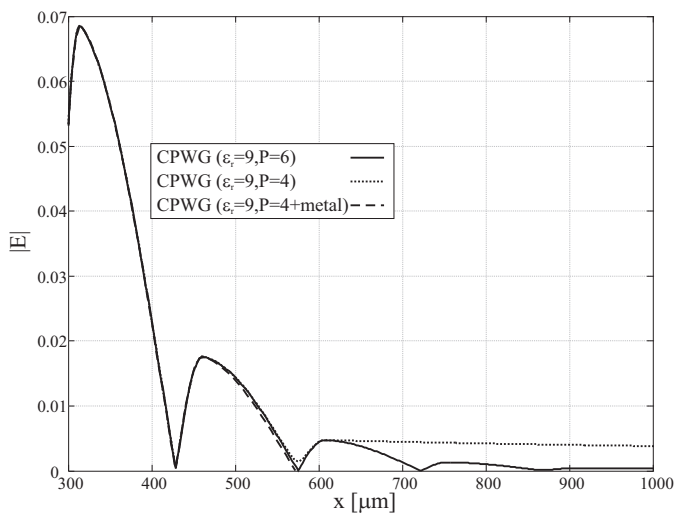

(b)

Fig.4 Electric field distribution (a) for several dielectric constants including HFSS simulations and (b) when two more layers are added and when the last layer is made of metal.

\section{Dielectric and Conductor Losses}

In the previous examples three loss contributions can be identified. If the layered structure is terminated by an open (air), there is both radiation and dielectric loss. If it is closed (metal wall) the radiation loss converts into a conduction loss. The conductor loss and dielectric loss can be calculated by using the conventional perturbation formulas [8]. 
Both of these contributions have been calculated for $\tan \delta=1 \mathrm{e}^{-4}$ and $\sigma=1 \mathrm{e}^{7} \mathrm{~S} / \mathrm{m}$ and are shown in Fig. 5. Increasing the number of layers does not alter the dielectric loss since it is dominated by the field distribution at the initial layers. However if a lower dielectric constant is used, e.g. $\varepsilon r=3.8$, the dielectric loss will be higher since less power is confided in the core. Alternatively, the metal loss depends on the number of layers as well as on their dielectric constant, since the magnetic field decays at each period. These loss values are much lower than the ones associated with a standard single mode CWG, which would have been on the order of $250 \mathrm{dBs} / \mathrm{m}$ for this wavelength.

\section{Conclusions}

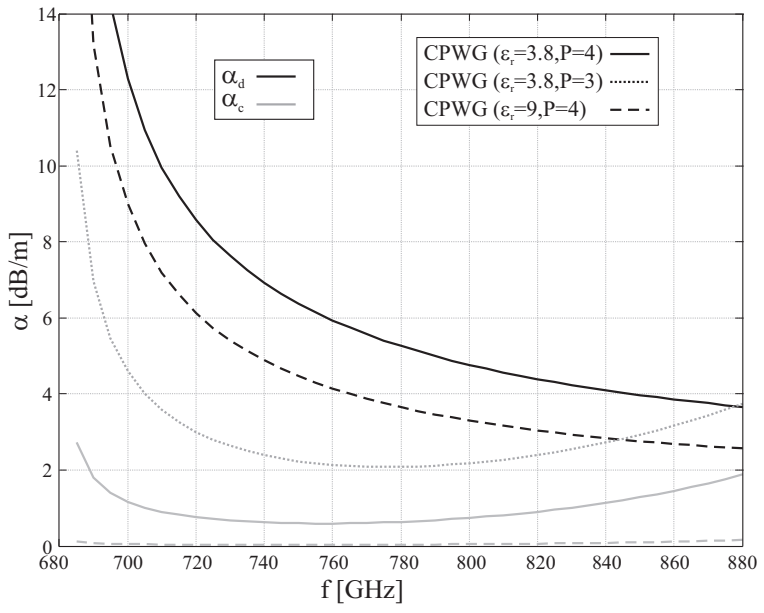

Figure 5: Dielectric and metal loss

In the terahertz band, the availability of dielectric materials for fabrication of long waveguides is very limited. The only viable candidate at the current time appears to be fused quartz which unfortunately has a relatively low dielectric constant. This limits the use of standard Brag fibers which rely on higher dielectric constants. To overcome this limitation, we have examined the possibility of using a small number of low dielectric constant layers in a periodic arrangement and enclosing the entire structure with a metalic boundary. This configuration results in a moderate loss waveguide. Future work will include the manufacture and testing of such structures and a study of the bend losses.

\section{References}

[1] C. Yeh, F. Shimabukuro, P. Stanton, V. Jamnejad, W. Imbriale, F. Manshadi; "Communication at millimeter-submillimetre wavelengths using a ceramic ribbon", Nature, vol. 404, April 2000.

[2] C. Yeh, F. Shimabukuro, P. H. Siegel, "Low-loss terahertz ribbon waveguides", Applied Optics, vol. 44, no.28, Oct. 2005.

[3] B. Temelkuran, S. D. Hart, G. Benoit, J. D. Joannopoulos, Y, Fink, "Wavelengthscalable hollow optical fibres with large photonic bandgaps for $\mathrm{CO} 2$ laser transmission", Nature, vol. 420, Dec. 2002

[4] M. Ibanescu, S. G. Johnson, M. Soljac 'ic', J. D. Joannopoulos, Y. Fink "Analysis of mode structure in hollow dielectric waveguide fibers", Physical Review E 67, 2003.

[5] D. R. Jackson, A. A. Oliner, and A. Ip, "Leaky wave propagation and radiation for a narrow-beam multiple layer dielectric structure," IEEE Trans. Antennas Propag., vol. 41, Mar. 1993.

[6] W. C. Chew, Waves and Fields in Inhomogenous Media, Van Nostrand Reinhold, 1990.

[7] P. Yeh, A. Yariv, "Theory of Bragg fibers”, J. Opt. Soc. Am., vol.68, no. 9, 1978.

[8] D. M. Pozar, Microwave Engineering, Wiley, 2005 